\title{
The Sir1 protein's association with a silenced chromosome domain
}

\author{
Kelly A. Gardner and Catherine A. Fox ${ }^{1}$ \\ Department of Biomolecular Chemistry, University of Wisconsin, Madison, Wisconsin 53706, USA
}

\begin{abstract}
Silencing of the cryptic mating-type locus $H M R$ requires recognition of a small DNA sequence element, the HMR-E silencer, by the Sir1p, one of four Sir proteins required for the assembly of silenced chromatin domains in Saccharomyces cerevisiae. The Sir1p recognizes the silencer through interactions with the origin recognition complex (ORC), a protein complex that binds the silencer DNA directly. Sir1p was physically associated with HMR in chromatin, and this association required a Sir1p-ORC interaction, suggesting that it reflected the Sir1p silencer-recognition function required for silencing. Sir1p was not associated with nonsilencer replication origins that bind the ORC, indicating that a Sir1p-ORC interaction is confined to silencers. Significantly, the other SIR genes were required for Sir1p's association with HMR. Thus, multiple protein contacts required for and unique to silent chromatin may confine a Sir1p-ORC interaction to silencers. The Sir1p was present at extremely low concentrations in yeast cells yet was associated with HMR at all stages of the cell cycle examined. These data provide insights into the mechanisms that establish and restrict the assembly of silenced chromatin to only a few discrete chromosomal domains.
\end{abstract}

[Key Words: Silencing; Sir; ORC; yeast; chromatin]

Received September 21, 2000; revised version accepted December 5, 2000.

Silencing is a form of transcriptional repression in yeast that requires the assembly of a repressive chromatin structure analogous to heterochromatin in multicellular eukaryotes (Hendrich and Willard 1995; Pillus and Grunstein 1995). Both heterochromatic and silenced domains contain relatively hypoacetylated histones that, in combination with specialized nonhistone chromatin-associated proteins, form a repressive chromatin structure recalcitrant to a variety of activities requiring access to the chromosomal DNA. These specialized chromatin domains can transcriptionally repress genes contained within them, but they also can influence several other aspects of DNA metabolism including DNA replication, recombination, and repair and chromosome stability (Gottlieb and Esposito 1989; Terleth et al. 1989, 1992; Freeman-Cook et al. 1999; Stevenson and Gottschling 1999; Henikoff 2000). Histone modifications and nonhistone proteins required for the assembly and maintenance of these specialized chromatin structures have been identified (Eissenberg et al. 1995; Hendrich and Willard 1995; Pillus and Grunstein 1995), but the mechanisms that target and confine the formation of repressive chromatin to specific domains within the eukaryotic genome are unclear.

Silencing of the cryptic mating-type loci, $H M R$ and $H M L$, in the yeast Saccharomyces cerevisiae is an ex-

${ }^{1}$ Corresponding author.

E-MAIL cfox@facstaff.wisc.edu; FAX (608) 262-5253.

Article and publication are at www.genesdev.org/cgi/doi/10.1101/ gad.852801. ample of the role repressive chromatin structures play in blocking transcription. Silencing of $H M R$ and $H M L$ is not required for yeast cell viability but is important in regulating haploid mating types by repressing transcription of cryptic copies of mating-type genes present at $H M R$ and $H M L$ (Herskowitz et al. 1992). Silencing at both loci requires the combined action of small DNA elements $(\sim 150 \mathrm{bp})$ called silencers, several multifunctional DNA-binding proteins that bind silencers directly (silencer-binding proteins), and the four specialized silencing-specific Sir (Silent Information Regulator) proteins (Loo and Rine 1995). The silencer-binding proteins are proposed to nucleate the assembly of a Sir protein complex(es) that encompass $\sim 4$-kb pairs of chromosomal DNA at the HMR and HML loci (Loo and Rine 1994; Triolo and Sternglanz 1996; Donze et al. 1999).

The recognition of the $H M R$-E silencer by the Sir 1 protein is proposed to be a key step in targeting the assembly of silenced chromatin to $H M R$. The $H M R$-E silencer contains binding sites for three multifunctional nuclear proteins: the origin recognition complex (ORC), Raplp, and Abflp. The ORC must bind to its site (an ACS) within $H M R$-E to function in silencing $H M R$ (McNally and Rine 1991; Foss et al. 1993; Micklem et al. 1993; Fox et al. 1995; Loo et al. 1995; Palacios DeBeer and Fox 1999). The available evidence indicates that the primary role for the ORC in silencing $H M R$ is recruitment of the Sirlp to $H M R$-E. In particular, the requirement for the ORC in silencing $H M R$ can be bypassed by tethering a Gal4-Sirlp fusion protein to the HMR-E silencer through a Gal4-binding site that replaces the silencer's ACS 
(Chien et al. 1993; Fox et al. 1997). This Gal4-Sirlptethered silencing requires the three other Sir proteins (Sir2p, Sir3p, and Sir4p), indicating that Sir1p can recruit the other Sir proteins to $H M R$ in the absence of an intact silencer (Chien et al. 1993). In addition, Sir1p and Orc1p, the largest subunit of the six-subunit ORC (Bell and Stillman 1992), interact directly (Triolo and Sternglanz 1996), and mutations in SIR1 that specifically abolish this interaction lead to defects in silencing (Gardner et al. 1999). These and additional observations have led to a model in which an ORC bound to a silencer recruits the Sirlp that in turn helps to recruit the other Sir proteins to the silent loci where they function as structural components of silenced chromatin. In this view, Sirlp has a regulatory role in the establishment of silenced chromatin but is less important to the inherent structure of silenced chromatin or its maintenance and inheritance once established (discussed in Loo and Rine 1995).

The $H M R$-E silencer also contains one binding site each for the Rap1p and Abf1p proteins, two abundant nuclear proteins with additional roles in telomere structure, transcription activation, and DNA replication and repair at other positions in the genome (Marahrens and Stillman 1992; Shore 1994; Kang et al. 1995; GailusDurner et al. 1996; Rolfes et al. 1997; Reed et al. 1999). Like ORC, both Raplp and Abflp must bind their sites within $H M R$-E to function in silencing. However, there is no evidence that either Raplp or Abflp functions in recruiting the Sirlp to $H M R$-E.

As is true for Raplp and Abflp, the ORC also has a role in addition to silencing. Specifically, the ORC is required for the initiation of DNA replication at each of the hundreds of chromosomal origins scattered throughout the yeast genome (Bell and Stillman 1992; Fox et al. 1995; Liang et al. 1995). The ORC's two different roles in yeast, silencing and DNA replication, both of which require its binding to an ACS, raise questions about the factors that govern a Sirlp-ORC interaction and the role this interaction plays in defining a silencer. In contrast with the ORC, the Sirlp functions only in silencing. Because the ORC binds to hundreds of positions in the yeast genome but only four of these positions correspond to known silencers, an important question is what factors distinguish an ORC bound to a silencer from an ORC bound to a nonsilencer replication origin. A prevalent but untested hypothesis is that Sirlp binds to silencer-bound ORC molecules but not to the ORC molecules bound to nonsilencer replication origins.

In this report, we addressed the current view of the Sirlp in silencing $H M R$. Chromatin immunoprecipitation experiments indicated that the Sirlp specifically associated with $H M R$ in chromatin, and that this association required the Sirlp-Orclp interaction. Significantly, Sirlp did not associate with nonsilencer replication origins, providing direct evidence that the SirlpORC interaction is a determinant that distinguishes a silencer from a nonsilencer replication origin. Interestingly, Sirlp's association with HMR required SIR2, indicating that other components of silenced chromatin may contribute to a stable Sirlp-ORC interaction at silencers. The Sirlp was present at very low concentrations in yeast, yet associated with $H M R$ throughout the cell cycle in a manner similar to Sir3p, a known structural component of silent chromatin. Taken together, these data put constraints on models for the role of Sirlp in the establishment of a silenced chromosome domain.

\section{Results \\ Sir1p was physically associated with $H M R$ in chromatin}

A current model for silencing $H M R$ posits that Sirlp binds to the $H M R$-E silencer in chromatin through interactions with the ORC that is bound directly to the silencer ACS. To test directly whether Sirlp is in close physical proximity to the $H M R$-E silencer DNA, we performed chromatin immunoprecipitation with antibodies against the hemagglutinin epitope ( $\alpha$-HA) by using yeast expressing a HA-tagged version of Sirlp (Sirlp-3xHA). The synthetic $H M R$-E silencer (HMR-SS) was used in most experiments because it lacks the redundancy present in the natural silencer and absolutely requires both Sirlp and individual binding sites within the silencer for silencing (McNally and Rine 1991).

If Sirlp was associated with HMR-SS in chromatin, then $H M R$-SS DNA should be enriched in a proteinDNA fraction prepared by immunoprecipitation of Sirlp-3xHA from a yeast whole cell extract. Sir1p-3xHA functioned indistinguishably from wild-type Sirlp in vivo (data not shown) and was detectable with an antibody to HA $(\alpha-\mathrm{HA})$ as an $\sim 85-\mathrm{kD}$ protein on an immunoblot (Fig. 1A). The smaller immunoreactive band visible in both lanes in this experiment was due to the immunoglobulin heavy chain from $\alpha$-HA. Yeast cells harboring a chromosomal copy of SIR1-3xHA were cross-linked with formaldehyde to create covalent linkages between closely associated proteins and proteins and DNA. The cross-linked chromatin was isolated and sheared, and Sirlp-3xHA was immunoprecipitated from this mixture by using $\alpha$-HA. The $H M R$-SS locus and the nonsilent $A D H 4$ or ACT1 locus were detected in the starting mixtures and in the immunoprecipitates with specific primers (Table 1) and PCR.

Significantly, HMR-SS was enriched in the immunoprecipitate, and this enrichment required both SIR1$3 x H A$ and $\alpha$-HA (Fig. 1B, cf. lanes 1-3). Importantly, this enrichment was specific for silencer DNA; the nonsilenced control locus $A D H 4$ was not enriched specifically in the immunoprecipitate (Fig. 1B, lanes 4-6). In separate experiments, the natural $H M R$-E silencer was specifically enriched in a Sirlp-3xHA-dependent chromatin immunoprecipitation experiment, indicating that Sirlp$3 \mathrm{xHA}^{\prime}$ 's behavior with the HMR-SS was an accurate reflection of natural Sirlp function, consistent with the previously published genetic analysis of this silencer (McNally and Rine 1991). 


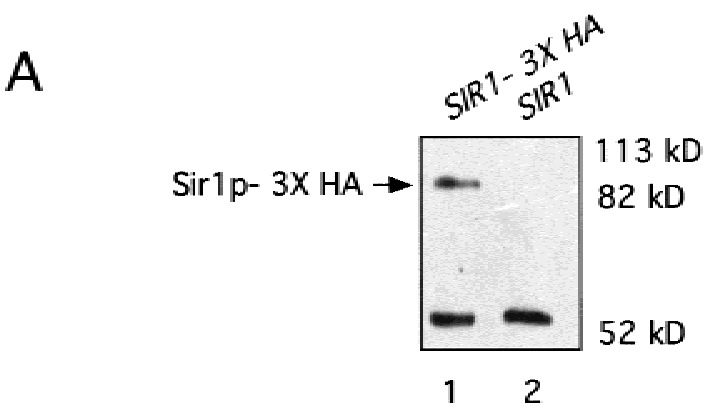

B

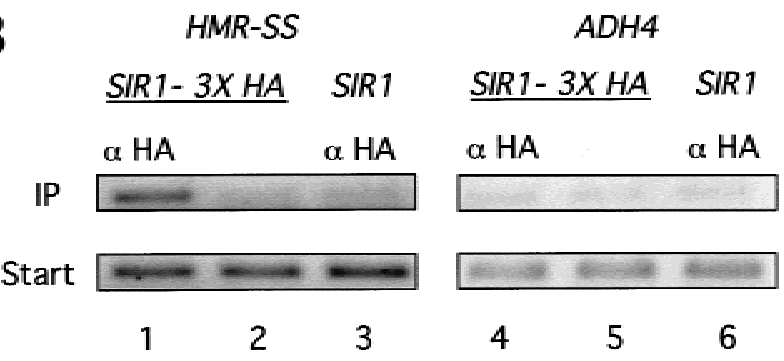

Figure 1. Sirlp was physically associated with $H M R$ in chromatin. (A) Protein immunoblot analysis of Sirlp-3xHA quantitatively immunoprecipitated with $\alpha$-HA. Twenty $\mathrm{A}_{600}$ cell equivalents of the immunoprecipitated fraction were analyzed in each lane. The smaller band seen in this immunoblot is due to binding of secondary antibody to the heavy chain of the $\alpha$-HA. $(B)$ Chemically cross-linked and sheared chromatin was immunoprecipitated with $\alpha$-HA from strains that expressed either SIR1-3xHA (lanes 1 and 4; CFY815) or untagged SIR1 (lanes 3 and 6; CFY345). HMR-SS DNA (lanes 1-3) and ADH4 DNA (lanes 4-6) were analyzed in the crude extract (Start) and immunoprecipitated (IP) fraction by using PCR directed by specific primers (Table 1). A mock precipitation of extracts from cells expressing SIR1-3xHA was performed that was identical to the $\alpha$-HA-directed immunoprecipitation except that it lacked $\alpha-\mathrm{HA}$ antibody (lanes 2 and 5).

Sir1p's association with $H M R$ required the silencer ORC-binding site and a region of Sir1p required for interaction with Orc1p

Several independent genetic experiments indicate that Sirlp's recognition of the HMR-E silencer in vivo requires the ORC bound to the silencer ORC-binding site (ACS). If Sirlp's physical association with $H M R$ observed above reflected this recognition, then the silencer ACS should be required for enrichment of $H M R$ in a Sirlp3xHA-dependent chromatin immunoprecipitation experiment. Therefore, we determined whether the association between Sirlp and $H M R$ was abolished by a mutation of this silencer's ACS (Fig. 2A). Significantly, in a strain containing a mutation of the ACS in the synthetic silencer (HMR-SS[acs-]), the silencer DNA was not enriched in the immunoprecipitate (Fig. 2A).

The Sirlp contains a small region that is dedicated to Sirlp's recognition of a silencer and required for Sirlp's interaction with the Orclp, the largest subunit of ORC (Gardner et al. 1999). Mutant Sirl proteins with single amino acid substitutions in this region function normally when tethered to the silencer through a heterologous DNA-binding domain but fail to silence $H M R$ by the normal mechanisms that presumably require Sirlp's physical association with the HMR-E silencer. These mutant Sirl proteins are referred to as silencer-recognition-defective $\left(\operatorname{Sirl} \mathrm{p}^{\mathrm{srd}}\right)$, and the region required for interaction with Orclp is referred to as the SRD region of Sirlp. To test whether the SRD region of Sirlp was required for Sirlp's ability to associate with HMR in chromatin, we examined whether two different mutant Sirlp ${ }^{\text {srd }}$ proteins enriched the HMR-SS DNA in a chromatin immunoprecipitation experiment (Fig. 2B). Both mutant Sirlp $p^{\text {srd }}$ proteins were expressed from the SIR1 locus as fusions to the $3 \mathrm{x}$-HA epitope (sir1-101-3xHA; sir1-102-3xHA). Notably, neither mutant Sirlp ${ }^{\text {srd }}$ enriched $H M R$-SS DNA as efficiently as wild-type Sirlp (Fig. 2B), indicating that this region of Sirlp was required for Sirlp's association with a silencer in chromatin. Importantly, the mutant proteins were expressed at levels equivalent to wild-type Sirlp, indicating that the inability of these mutant proteins to enrich silencer DNA in the immunoprecipitate was not due to significant reductions in the levels of the mutant Sirl ${ }^{\text {srd }}$ proteins (Fig. 2C).

Taken together, these data indicate that Sirlp is physically associated with $H M R$ in chromatin and support the hypothesis that this association is required for Sirlp's function in silencing.

The Sir1p did not associate with nonsilencer origins If a Sirlp-silencer interaction were a key determinant of

Table 1. Oligonucleotide primer pairs used in this study

\begin{tabular}{|c|c|c|}
\hline \multirow{2}{*}{$\frac{\text { Locus }}{A D H 4}$} & \multicolumn{2}{|c|}{ Primer pair } \\
\hline & ggctactaacggtggggaaatcggagac & gcacaggcatcggtgattgggttagaggc \\
\hline$A C T 1$ & cggtattgtcaccaactgggacgatatgg & gcagcggtttgcatttcttgttcgaagtcc \\
\hline ARS306 & ctgtagtttggacaaggtgaccetgccaag & gtgacttactaacgtcaacgtacaatcgcg \\
\hline$A R S 1$ & ggcgttattggtgttgatgtaagcggagg & gcaagaccgagaaaaggctagcaagaatcg \\
\hline ARS1412 & gggaatagctaatcaagtggataagacgc & ccaactcctctctacttgcgtgtgtatttg \\
\hline$H M R(\mathrm{a})$ & ctaacttcaactgttgctggtgtcgcetgc & gcccatttcgaagaatgattgagcaccgtc \\
\hline$H M R(\mathrm{~b})$ & caagggcctacgattactatgtactggaagg & ggtcaacataaagtggcgagaaaaacgccetg \\
\hline$H M R$-SS & ggtagagttccttgttgaacgtgataaccc & gatgtctgggtttgtttggcatgcatcagc \\
\hline$H M R(\mathrm{~d})$ & cttcttctgttgttacactctctggtaacttagg & cetgttctaaaaatgccegtgcttggggtg \\
\hline$H M R(\mathrm{e})$ & caaactttgagagaaatatgtctttctactgcg & cctaccacattatcaatccttgcatccagc \\
\hline$H M R(\mathrm{f})$ & gccaacaatggaatctcattacccatcccaag & gtactcacacctttgcaagactaccaggatc \\
\hline$H M R(\mathrm{~g})$ & gatatcgccactgcatcatttctgtagtcg & cagtcagacaggtcgatcaaagttgaaattgg \\
\hline
\end{tabular}


silent chromatin assembly at $H M R$, then Sirlp should not associate with nonsilencer origins even though these elements bind the ORC. Therefore, we tested whether several characterized replication origins were associated with Sirlp by using chromatin immunoprecipitation

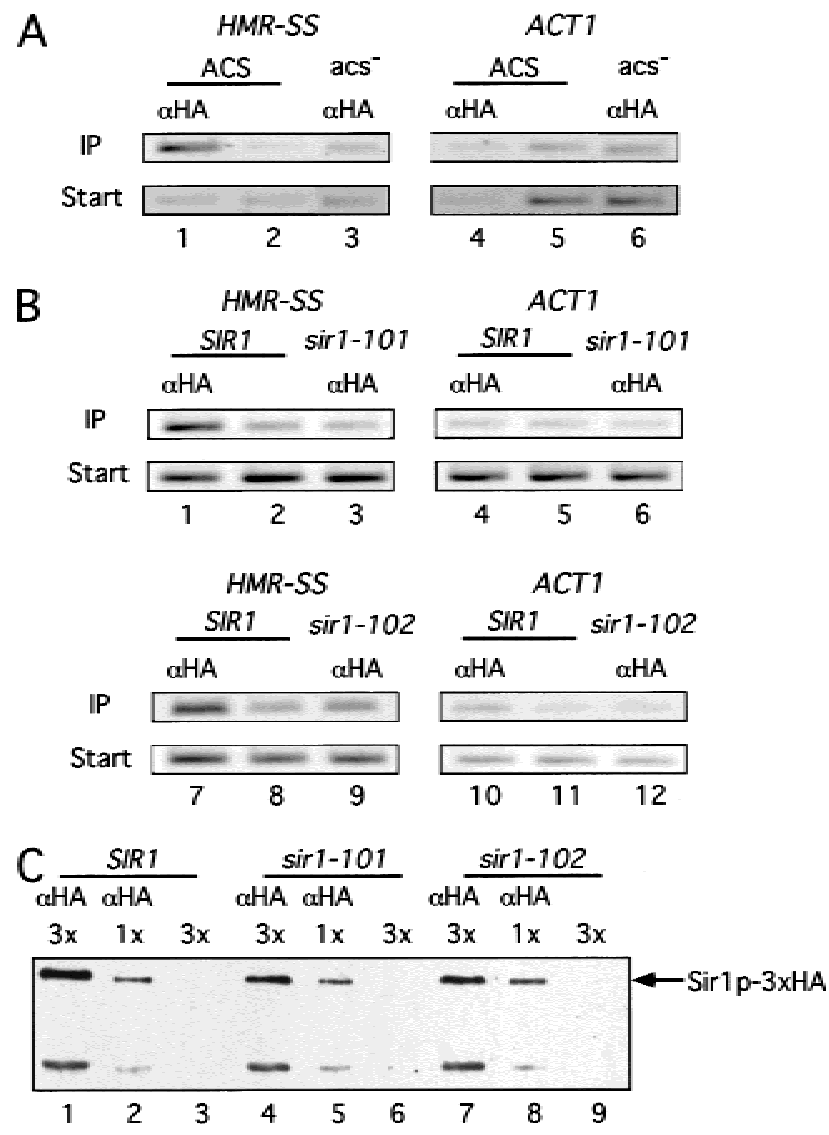

Figure 2. Sirlp's association with $H M R$ required the silencer ACS and a region of Sirlp required for interaction with Orclp. The chromatin immunoprecipitation experiments were performed as described in Fig. 1 except that ACT1 was used as a nonsilenced control locus. (A) A mutation of the silencer ACS abolished Sirlp's association with $H M R$. Chromatin immunoprecipitation of $H M R$-SS was compared for two isogenic strains that contained either wild-type HMR-SS (ACS; CFY476; lanes 1 and 2 and 4 and 5) or HMR-SS containing a mutation in the ACS (HMR-SS [acs-]) (acs ${ }^{-}$; CFY633; lanes 3 and 6). (B) A mutation in the region of Sirlp required for interaction with Orclp abolished Sirlp's association with $H M R$. Chromatin immunoprecipitation of $H M R$-SS was compared for three isogenic strains that contained either wild-type SIR1-3xHA (SIR1; CFY815; lanes 1 and 2, 4 and 5, 7 and 8 , and 10 and 11) or one of two $\operatorname{sir} 1^{\text {std }}$ $3 \times H A$ alleles (sir1-101; CFY689; lanes 3 and 6; sir1-102; CFY687; lanes 9 and 12) at the chromosomal SIR1 locus. (C) The mutant Sir $1^{\text {srd }}-3 \mathrm{xHA}$ proteins were expressed at the same levels as wild-type Sirlp-3xHA. Protein immunoblot analysis of wildtype and mutant Sirlp-3xHA proteins quantitatively immunoprecipitated with $\alpha$-HA from the strains used in the experiment described above $(B)$. Protein was analyzed in the immunoprecipitated fractions of extracts prepared from $45 \mathrm{~A}_{600}(3 \times)$ (lanes $1,4,7)$ or $15 \mathrm{~A}_{600}(1 \times)$ (lanes 2,5,8) cell equivalents. Mock precipitations were performed as described in Fig. 1 (lanes 3, 6, and 9).
(Fig. 3; data not shown). In these experiments, Sirlp associated with the $H M R$-E silencer within chromatin but not with other origins including an early (ARS306), middle $(A R S 1)$, and late $(A R S 1412)$ replication origin. In addition, Sirlp did not associate with several different inefficient origins on chromosome VI, nor did it associate with natural telomeric origin sequences /data not shown). Thus, although the available evidence supports only a direct interaction between Sirlp and Orclp, other factors in addition to ORC contribute to Sirlp's association with $H M R$ in chromatin.

The other SIR genes were required for the association of Sir1p with HMR

The HMR-E silencer contains binding sites for two other silencer-binding proteins in addition to the ORC, specifically the Raplp and the Abflp, that could contribute to Sirlp's preference for a silencer over nonsilencer origins. Therefore, we tested whether the Rap1p or Abf1p binding sites were required for enrichment of the HMR-SS (Fig. 4). Significantly, in a strain containing a mutation of the Raplp-binding site in the synthetic silencer (HMR$\left.\mathrm{SS}\left[\operatorname{rap} 1^{-}\right]\right)$, Sirlp-3xHA did not associate with the HMRSS (Fig. 4A). In contrast, an analogous experiment demonstrated that the Abflp-binding site was not required for Sirlp-3xHA's association with HMR-SS in chromatin (Fig. 4B).

A crucial role of the Raplp in silencing at both telomeres and the HM loci is to recruit the Sir2/Sir3/Sir4 protein complex that functions in the assembly and maintenance of silent chromatin (Moretti et al. 1994; Grunstein 1997). Thus, the requirement for the silencer's Raplp-binding site in Sir1p-3xHA's association with $H M R$-SS could actually reflect a requirement for the other Sir proteins. Therefore, we tested whether Sirlp$3 \mathrm{xHA}$ associated with HMR-SS in yeast cells harboring a deletion of SIR2 (Fig. 5). Significantly, in strains lacking SIR2, Sirlp-3xHA failed to enrich for HMR-SS (Fig. 5A). The nuclear concentration of Sirlp-3xHA was not reduced in sir2 $\Delta$ mutant strains, indicating that the lack of a Sirlp-3xHA-silencer interaction in chromatin in this mutant was not due to a lack of nuclear Sir1p-3xHA (Fig. 5B). In separate experiments, a sir3 $\Delta$ mutant behaved similarly (data not shown). Thus, the other SIR genes are required for a stable association between the Sirlp and $H M R$ in chromatin.

\section{The Sir1p and Sir3p associated similarly with HMR}

A prevalent view of Sirlp in silencing $H M R$ differs considerably from the view of Sir3p. In particular, the Sirlp, a proposed regulator of silenced chromatin assembly (Pillus and Rine 1989; Loo and Rine 1995; Triolo and Sternglanz 1996), might be expected to associate with $H M R$ differently from Sir3p. Therefore, we compared the association of Sirlp and Sir3p with HMR in a chromatin immunoprecipitation experiment (Fig. 6).

To compare the association of Sirlp and Sir3p with 
Figure 3. The Sirlp was not associated with nonsilencer origins. Chromatin immunoprecipitation with $\alpha$-HA was performed using a strain expressing SIR13xHA (CFY815; lanes 1,3,5,7,9). The appropriate primers (Table 1) were used to examine the Sirlp's association with
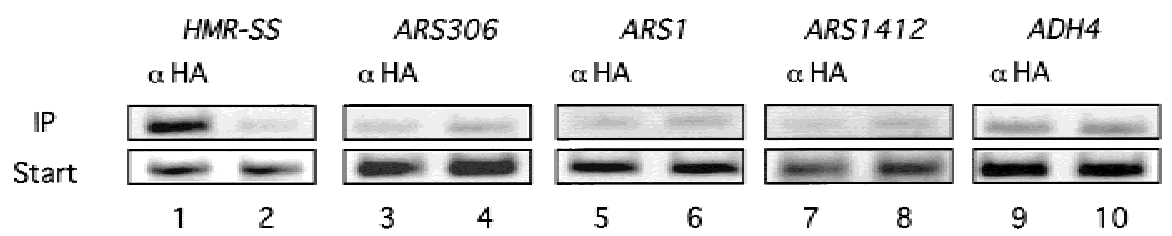

HMR-SS and several nonsilencer origins as indicated. A mock precipitation was performed as described in Fig. 1, and the precipitate was examined for DNA with the same primers (lanes 2,4,6,8,10).

$H M R$, chromatin immunoprecipitation experiments were performed with two isogenic strains that differed in the form of Sir protein that was expressed as an HAtagged protein. Specifically, one strain expressed Sirlp$3 \mathrm{xHA}$ and the second strain expressed Sir3p-3xHA. After chromatin isolation, the sheared chromatin was immunoprecipitated from each strain, and different regions of $H M R$ were examined using the appropriate PCR primers as diagrammed in Figure 6 and listed in Table 1.

In three independent experiments, one of which is shown in Figure 6, the association of Sirlp and Sir3p with $H M R$ was virtually identical. Although the enrichment of the most outlying $H M R$ fragments varied somewhat in individual experiments, the behavior of Sirlp$3 \mathrm{xHA}$ and Sir3p-3xHA was comparable. For example, in the experiment shown, fragment $b$ was not associated

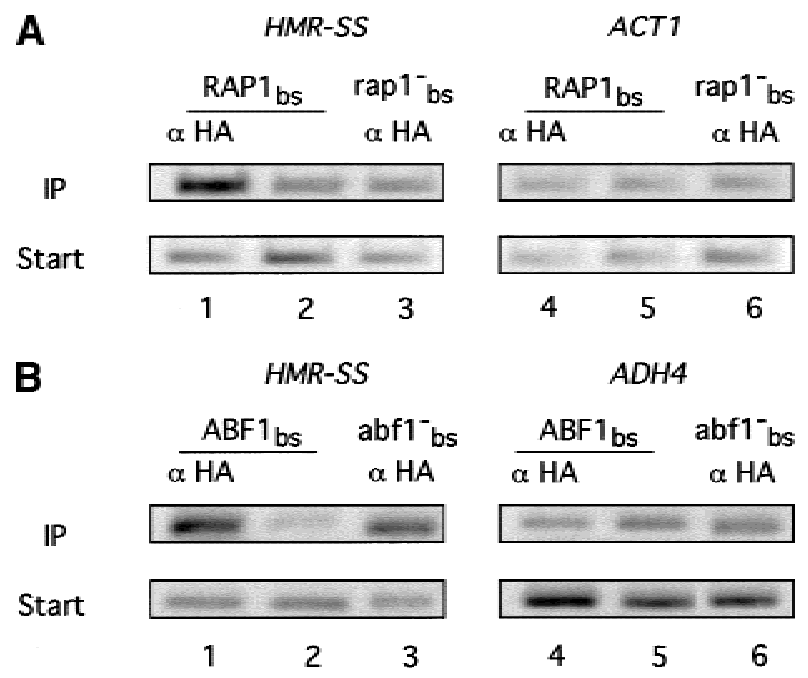

Figure 4. The silencer Raplp-binding site, but not the Abflpbinding site, was required for Sirlp's association with $H M R$. Chromatin immunoprecipitation was performed as described in Fig. 1. (A) A mutation of the silencer Raplp-binding site abolished Sirlp's association with $H M R$. Chromatin immunoprecipitation of $H M R$-SS was compared for two isogenic strains that contained either wild-type $H M R$-SS $\left(\mathrm{RAP}_{\mathrm{bs}}\right.$ CFY476; lanes 1 and 2 and 4 and 5) or HMR-SS containing a mutation in

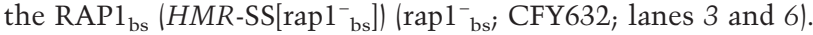
(B) A mutation of the silencer Abf1p-binding site did not abolish Sirlp's association with $H M R$. Chromatin immunoprecipitation of $H M R$-SS was compared for two isogenic strains that contained either wild-type $H M R$-SS $\left(\mathrm{ABF}_{\mathrm{bs}}\right.$; CFY476; lanes 1 and 2 and 4 and 5) or $H M R$-SS containing a mutation in the $\mathrm{ABF}_{\mathrm{bs}}\left(H M R\right.$-SS[abf1 $\left.\left.{ }^{-} \mathrm{bs}\right]\right)\left(\mathrm{abf1}{ }^{-}{ }_{\mathrm{bs}}\right.$ CFY625; lanes 3 and 6). with either Sir1p-3xHA or Sir3p-3xHA based on chromatin immunoprecipitation. In other experiments, fragment b was associated with both Sirlp-3xHA and Sir3p$3 x H A$. Thus, at the low resolution offered by these experiments, Sirlp-3xHA and Sir3p-3xHA associated similarly with $H M R$.

The Sir1p was expressed at low levels in yeast cells and was associated exclusively with a chromatincontaining fraction

The data described above supported the view that a Sirlp-ORC interaction is confined to silenced regions such as $H M R$. Assuming that a Sirlp-ORC interaction is stable, one factor that could help confine it to silenced regions is a low concentration of Sirlp in yeast. Therefore, we determined the levels of Sirlp-3xHA expressed per yeast cell by comparing the amount of Sirlp-3xHA in a known number of yeast cells to a $3 x H A$-containing standard protein of known concentration (Fig. 7). In one

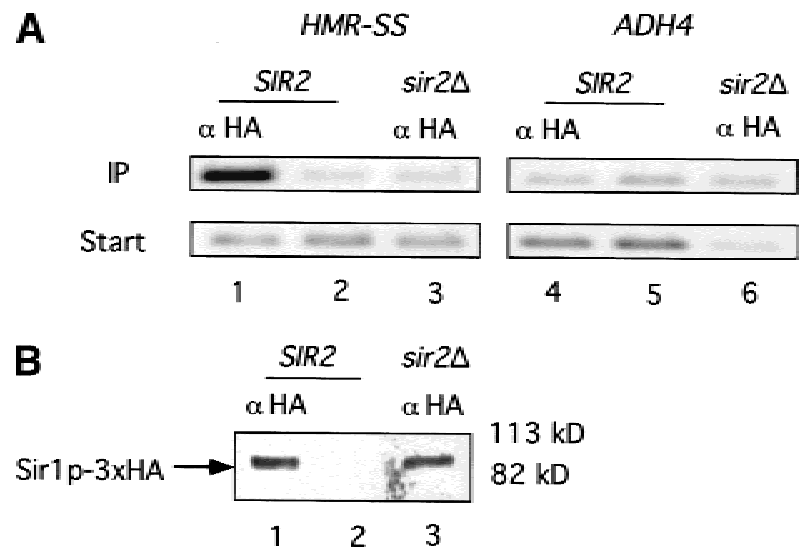

Figure 5. SIR2 was required for Sirlp's association with $H M R$ in chromatin. Chromatin immunoprecipitations were performed as described in Fig. 1. (A) A sir2s mutation abolished Sirlp's association with $H M R$. Chromatin immunoprecipitation of $H M R$-SS was compared in two isogenic strains that contained SIR1-3xHA and either a wild-type copy of SIR2 (CFY815; SIR2; lanes 1 and 2 and 4 and 5) or a deletion of SIR2 (sir2A; CFY105; lanes 3 and 6). (B) The nuclear concentration of Sirlp3xHA was not reduced by a mutation in SIR2. Sirlp was immunoprecipitated from nuclear extracts prepared from isogenic strains that each contained SIR1-3xHA and either wild-type SIR2 (CFY815; lanes 1 and 2 and lanes 4 and 5) or sir2A (CFY105; lanes 3 and 6). 

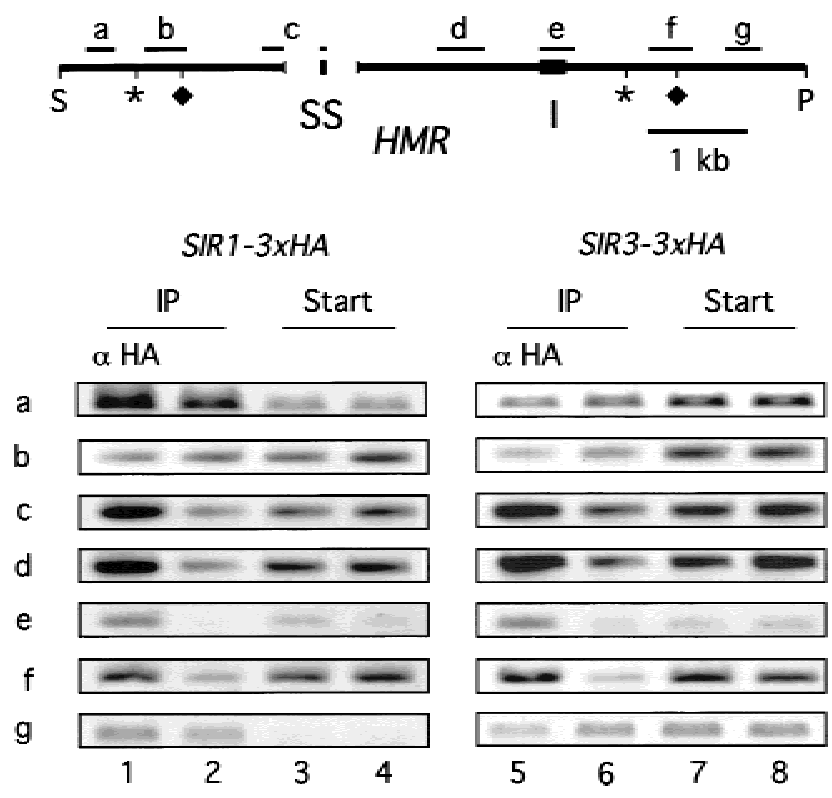

Figure 6. The Sirlp and Sir3p associated similarly with $H M R$. Chromatin immunoprecipitations were performed as described in Fig. 1 by using isogenic strains expressing either SIR1-3xHA (CFY762 containing pRS316-SIR1-3xHA [pCF448]; lanes 1 and 4) or SIR3-3xHA (CFY914 containing pRS316-SIR3-3xHA [Ansari and Gartenberg 1999]; lanes 5 and 8). The presence of particular fragments within and surrounding $H M R$ in the immunoprecipitates was determined using specific primers diagrammed in the figure and listed in Table 1. (S) Sph1 site on chromosome III at Saccharomyces Genome Database (SGD) coordinate 289759; (P) Pvul site at SGD coordinate 297608. The gap in the line represents a deletion of $\sim 800$ bp that includes the $H M R$-E silencer and flanking sequences; these deleted sequences were replaced with the HMR-SS (McNally and Rine 1991). One primer of the pair used to analyze fragment c within $H M R$ annealed to sequences within $H M R$-SS, and the second primer annealed to sequences flanking the 800-bp deletion, which is why fragment $\mathrm{c}$ is depicted as "interrupted." (Asterisks and diamonds) Boundaries of silenced chromatin as determined by Loo and Rine (1994) and Donze et al. (1999), respectively. The sequences of all primers used to generate the fragments indicated in the figure are listed in Table 1.

approach, Sir1p-3xHA was quantitatively immunoprecipitated from $30 \mathrm{~A}_{600}$ cell equivalents of a denatured crude yeast cell extract, and known amounts of a purified standard protein (GST-Ssq1p-3xHA; Voisine et al. 2000) were added to the immunoprecipitate before gel electrophoresis (Fig. 7A). Importantly, Sir1p-3xHA could not be detected in a second immunoprecipitation of the supernatant that remained after the first immunoprecipitation, suggesting that the Sirlp-3xHA had been quantitatively immunoprecipitated (K.A. Gardner, data not shown). Moreover, digestion of the yeast whole cell extract with DNAse and/or treatment with denaturing detergents did not change the amount of Sirlp-3xHA that could be immunoprecipitated (K.A. Gardner, data not shown). These experiments indicated that the Sirlp$3 x H A$ was present at a concentration of $\sim 10-30 \mathrm{~mol}$ ecules per cell, with the precise number varying between individual experiments.
As a second independent method for measuring the concentration of Sirlp-3xHA that did not rely on an immunoprecipitation step, a crude yeast extract was separated into chromatin and soluble fractions, and the level of Sirlp-3xHA in these fractions was determined (Fig. 7B). Most of the Sir1p-3xHA was present in the chromatin pellet; Sirlp-3xHA could not be detected in the soluble fraction even after immunoprecipitation before protein immunoblot analysis (data not shown). The concentration of Sirlp-3xHA in a yeast cell based on the amount present in the chromatin fraction was $\sim 20$ molecules, a value close to that determined by quantitative immunoprecipitation of Sirlp-3xHA from a denatured crude extract (Fig. 7A). Therefore, only a few Sirlp molecules are present in a yeast cell, and most of these molecules are associated with a chromatin-containing fraction.

The Sir1p was associated with $H M R$ at the $G_{1}, S$, and $M$ phases of the cell cycle

Sirlp has been postulated to function in the S-phase requirement for the establishment of silenced chromatin at the HM loci (Miller and Nasmyth 1984; Pillus and Rine 1989, Loo and Rine 1995; Triolo and Sternglanz 1996; Fox et al. 1997). One intriguing possibility raised by this postulate is that an S-phase requirement for establishment could be accomplished, at least in part, by confining an ORC-Sirlp interaction at $H M R$ to the S phase of the cell cycle (Triolo and Sternglanz 1996). Therefore, we tested whether Sirlp-3xHA was associated with $H M R$ in chromatin at three different stages of the cell cycle by performing Sirlp-3xHA-dependent chromatin immunoprecipitation experiments in yeast cells arrested in either the $G_{1}, S$, or $M$ phase of the cell cycle (Fig. 8). Notably, the Sir1p-3xHA associated with $H M R$ at each stage of the cell cycle as efficiently as it associated with $H M R$ in the same yeast strain growing asynchronously (Fig. 8). Therefore, if Sirlp's role in silencing is confined to $S$ phase, this cell cycle regulation is not exerted at the level of Sirlp's physical association with $H M R$ in chromatin.

\section{Discussion}

\section{A Sir1p-ORC interaction in defining a silencer}

Several independent studies support the conclusion that a Sirlp-ORC interaction is critical for Sirlp's function in silencing HMR (Chien et al. 1993; Triolo and Sternglanz 1996; Fox et al. 1997; Gardner et al. 1999). The work presented here extends this conclusion by demonstrating directly that the natural Sirlp expressed from its normal locus physically associates with $H M R$ in chromatin in a manner that requires a Sirlp-ORC interaction. Moreover, this study provides evidence that a Sirlp-ORC interaction is a molecular feature that distinguishes a silencer from a nonsilencer replication origin because a Sirlp-ORC interaction could not be detected at several different nonsilencer origins.

Two specific and interdependent findings reported 
A

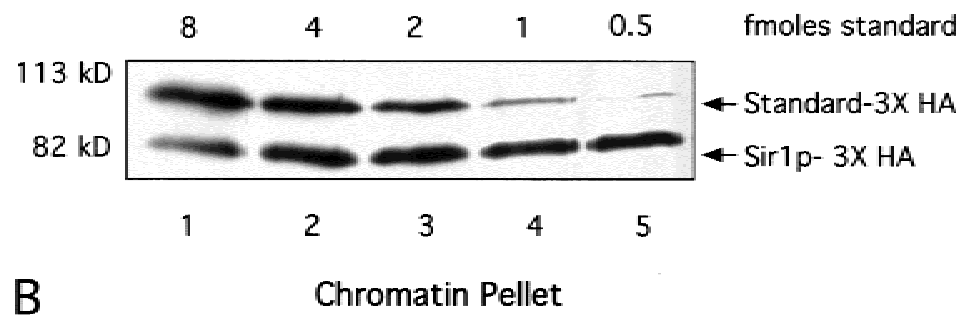

Figure 7. The Sirlp was expressed at low levels and was associated exclusively with a chromatincontaining fraction. (A) Quantitative immunoprecipitation of Sirlp-3xHA from yeast containing SIR1-3xHA at the chromosomal SIR1 locus (CFY815) indicated that Sirlp-3xHA concentration was $\sim 20$ molecules per yeast cell. Sirlp-3xHA was quantitatively immunoprecipitated from crude yeast extracts and $5.5 \mathrm{~A}_{600}$ cell equivalents of the immunoprecipitated fraction were mixed with decreasing amounts of a standard protein (standard-3xHA; purified and quantified Ssq1p-3xHA [Voisine et al. 2000]). (B) Sirlp-3xHA was present exclusively in a chromatin-containing fraction; $0.85 \mathrm{~A}_{600}$ cell equivalents of the chromatin-containing fraction were mixed with decreasing amounts of a standard protein (Ssq1-3xHA) as described in (A). Sirlp-3xHA could not be detected in the soluble fraction even after immunoprecipitation and protein immunoblot analysis. here contribute insights into the mechanisms that could confine a Sirlp-ORC interaction to silenced chromatin. First, the low concentration of Sirlp and its association with $H M R$ at various stages of the cell cycle are consistent with a stable Sirlp-ORC interaction within silenced chromatin domains. Such stability would limit the opportunity for Sirlp to interact with nonsilencer ORC molecules within the genome, a possibly important limitation considering Sirlp's role in assembling repressive chromatin and the discovery that at least one Sir protein can modulate the activity of replication origins (Stevenson and Gottschling 1999). Second, the requirement for the other SIR genes in Sirlp's association with $H M R$ indicates that the other Sir proteins, known structural components of silenced chromatin, may stabilize a Sirlp-ORC interaction that by itself may be inherently weak. Notably, a Sirlp-Sir4p interaction has been observed (Triolo and Sternglanz 1996), indicating that Sirlp does indeed contact at least one other Sir protein directly that could function in this way. Thus, multiple, and possibly individually relatively weak, interactions between Sir proteins and between Sir proteins and silencer-binding proteins may contribute to the ultimate stability of a Sirlp-ORC interaction in silenced chromatin, and this interaction in turn could enhance the stability of the silenced chromatin of which it is a part. The inference that additional proteins that comprise silent chromatin contribute to the stability and specificity of a Sirlp-ORC interaction underscores the probability that the assembly of large chromosomal regions into heritable transcriptional states involves a high degree of molecular cooperativity.

\section{The establishment and maintenance of a silenced chromatin domain}

Analysis of silencing during the cell cycle (Miller and Nasmyth 1984), and at the single-cell level (Pillus and Rine 1989; Mahoney et al. 1991), helped formulate the hypothesis that the Sirlp and silencers play regulatory roles in silencing. According to this hypothesis, the Sirlp is required for the establishment of silenced chromatin during the $S$ phase of the cell cycle but not the subsequent maintenance or propagation of the silenced state (discussed in Fox et al. 1993; Loo and Rine 1995). In addition, this Sirlp/S-phase-dependent establishment is required in only a few cell divisions to switch a cell from the nonsilenced to silenced state. The demonstration that Sirlp mediates its role in silencing through the silencers refined the view of Sirlp-mediated establishment (Chien et al. 1993). Specifically, establishment of silencing at $H M R$ often is depicted as the Sirlp binding to

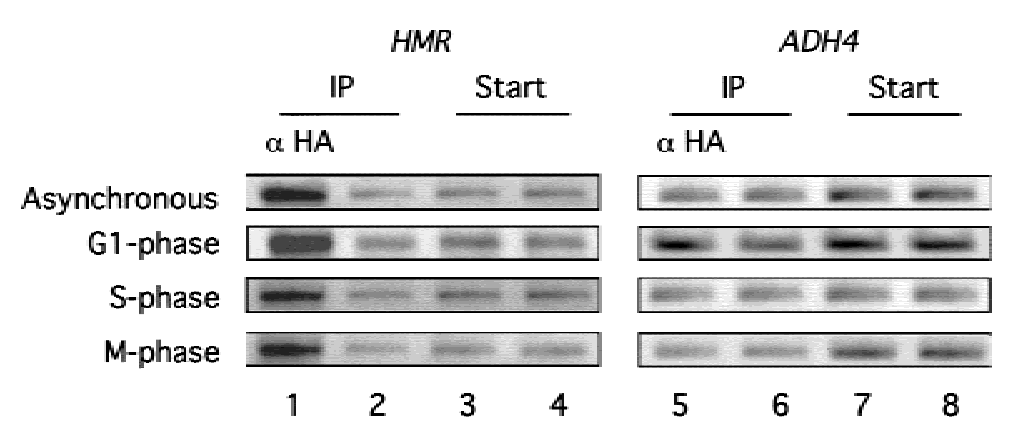

Figure 8. The Sirlp was associated with $H M R$ at the $\mathrm{G}_{1}, \mathrm{~S}$, and $\mathrm{M}$ phases of the cell cycle. Chemically crosslinked and sheared chromatin was immunoprecipitated with $\alpha$-HA (lanes 1 and 5) from a MATa HMRa strain that expressed SIR1-3xHA (CFY416) that was growing asynchronously or arrested with $\alpha$-factor $\left(\mathrm{G}_{1}\right.$ phase), hydroxyurea (S phase), or nocodazole (M phase). The natural HMR locus (lanes 1-4) and ADH4 DNA (lanes 5-8) were analyzed in the crude extract (Start) and immunoprecipitated (IP) fractions by using PCR directed by specific primers. A mock precipitation of extracts was performed that was identical to the $\alpha$-HA-directed immunoprecipitation except that it lacked $\alpha-\mathrm{HA}$ antibody (lanes 2 and 6). 
silencers in S phase through interactions with the ORC and nucleating the assembly of silenced chromatin by recruiting a complex consisting of the other Sir proteins (Triolo and Strenglanz 1996; Gardner et al. 1999). If establishment is required only rarely, Sirlp would need to "nucleate" silencing only once every several S phases because, once assembled, the silenced state self-propagates efficiently (Fox et al. 1993). In contrast with this view of Sirlp, the other Sir proteins are viewed as being important for maintaining the inherent structure of silenced chromatin throughout the cell cycle. Indeed, this view for the Sir2/3/4 proteins is supported by the important observation that these proteins are structural components of silenced chromatin (Hecht et al. 1995, 1996; Strahl-Bolsinger et al. 1997).

The data presented here put constraints on the mechanisms by which Sirlp could function in establishing silenced chromatin and highlight the possibility that Sirlp may have an equally important role in maintaining silenced chromatin throughout the cell cycle. In particular, the demonstration that the Sirlp is associated with $H M R$ throughout the cell cycle makes it improbable that a potential S-phase role for Sirlp in silencing is due to confining a Sirlp-ORC interaction to $S$ phase (Triolo and Sternglanz 1996). Moreover, the cell cycle data presented here are equally consistent with Sirlp having a "structural" role in silent chromatin at $H M R$, similar to the other Sir proteins. It is possible, for example, that one or more Sirlp molecules recognize ORC(s) within previously assembled, and therefore probably condensed, silenced chromatin and, by binding to these ORC molecules and other Sir proteins, structurally stabilize this chromatin state. If Sirlp does recognize and stabilize previously assembled and condensed silenced chromatin at $H M R$, only a few Sirl protein molecules would be necessary to "contact" the entire domain. Thus, a molecular component of SIR1-dependent establishment may be Sirlp's recognition and stabilization of previously assembled silenced chromatin. In this view, the establishment and stable propagation of silenced chromatin in a wild-type yeast population are not mechanistically distinct events. Rather than nucleating a new silenced state, Sirlp simply enhances the already relatively efficient process of self-propagation of a previously assembled silenced state. This view, although different from models for Sirlp function proposed previously, is entirely consistent with genetic data concerning the role of SIR1 and silencers in the establishment of silencing at the HM loci (Pillus and Rine 1989; Mahoney et al. 1991). Because Sirlp functions, at least in part, through the silencers, this view of Sirlp is also consistent with recent elegant molecular experiments that indicate that the silencers are required to maintain silencing throughout the cell cycle (Holmes and Broach 1996; Cheng and Gartenberg 2000).

There are many possible mechanisms by which a Sirlp-ORC interaction could enhance the stability of silenced chromatin and restrict it to a few discrete domains in the genome. As discussed above, the SirlpORC interaction could prevent the inherent instability of Sir2/3/4 silencing (Pillus and Rine 1989; Gottschling et al. 1990; Aparicio et al. 1991) by a direct structural mechanism. Alternatively, Sirlp could function as an enzyme that modifies a component of silenced chromatin, perhaps ORC itself, and this modification in turn could play a stabilizing structural role. The low concentration of Sirlp and the recent exciting data demonstrating that yeast Sir2p is an enzyme make this possibility worth considering (Tanney et al. 1999; Imai et al. 2000; Landry et al. 2000; Smith et al. 2000).

Although there are no known homologs of SIR1, it is probable that interactions between ORC and heterochromatin-associated proteins with roles analogous to yeast Sirlp will be important for regulating silencing in all eukaryotic organisms. In particular, the association of the Drosophila HP1 protein with heterochromatin requires an HP1-ORC interaction reminiscent of the Sirlp-ORC interaction in yeast (Pak et al. 1997; Huang et al. 1998), and, significantly, a human homolog of HP1 has been proposed to silence genes involved in breast cancer metastasis (Kirschmann et al. 2000). Given that ORC homologs have been identified in a variety of metazoans including humans (Quintana and Dutta 1999), it will be interesting to learn whether the function of this human HP1 homolog is mediated through interactions with the human ORC. Regardless of the exact mechanism by which Sirlp contributes to silencing, a comprehensive analysis of the Sirlp-ORC interaction in yeast undoubtedly will provide mechanistic insights relevant to the role of the ORC in chromosome structure and gene silencing in all eukaryotes.

\section{Materials and methods}

\section{Yeast strains}

The yeast strains used in this study are listed in Table 2 and were constructed using standard yeast molecular genetic techniques (Guthrie and Fink 1991). Chromosomal SIR1-3xHA was constructed using a PCR-mediated epitope-tagging method (Schneider et al. 1995). pRS316-containing SIR1-3xHA was constructed using a C-terminal SIR1-3xHA fragment derived from the chromosomal SIR1-3xHA. pRS316-containing SIR3-3xHA was provided by Marc Gartenberg (Ansari and Gartenberg 1999).

\section{Immunoprecipitation and immunoblotting}

To prepare extracts for analysis of Sirlp, the appropriate yeast cells were grown to a concentration of $2 \times 10^{7}$ cells $/ \mathrm{mL}$. The cells were harvested by centrifugation, washed twice with TBS (20 $\mathrm{mM}$ Tris- $\mathrm{HCl}$ at $\mathrm{pH} 7.5,200 \mathrm{mM} \mathrm{NaCl}$ ), and resuspended in $400 \mu \mathrm{L}$ FA-LB $150 \mathrm{mM}$ Hepes-KOH at pH 7.5, $140 \mathrm{mM} \mathrm{NaCl}, 1$ mM EDTA, $0.5 \%$ Triton X-100, 0.1\% sodium deoxycholate, and 1:500 dilution of CalBiochem protease inhibitor cocktail). The resuspended cells were mechanically broken by bead beating. To immunoprecipitate Sir1p-3xHA, we incubated anti-HA antibodies (BAbCo) with the extract overnight at $4^{\circ} \mathrm{C}$, and the extract/antibody mixture then was incubated an additional 2-6 h with $200 \mu$ 50:50 slurry of protein A-Sepharose CL-4B beads (Pharmacia). Immunoprecipitates were washed with $1 \mathrm{~mL}$ of each of the following: FA-LB, FA-LB/0.5M NaCl, wash $3(10 \mathrm{mM}$ Tris- $\mathrm{HCl}$ at $\mathrm{pH} 8.0,0.25 \mathrm{M} \mathrm{LiCl}, 0.5 \% \mathrm{NP}-40,0.5 \%$ sodium 
Table 2. Strains used in this study

\begin{tabular}{|c|c|}
\hline Strain & Genotype \\
\hline JRY3009 & $\begin{array}{l}\text { MAToade2-1 his3-11,15 leu2-3,112 trp1-1 ura3-1 } \\
\text { can1-100 (W303-1B) }\end{array}$ \\
\hline CFY345 & JRY3009 HMR-SS \\
\hline CFY815 & JRY3009 HMR-SS SIR1-3xHA \\
\hline CFY416 & JRY3009 MATa SIR1-3xHA \\
\hline CFY434 & JRY3009 MATa HMR-SS \\
\hline CFY419 & JRY3009 MATa HMR-SS(acs-) \\
\hline CFY423 & JRY3009 MATa HMR-SS(rap1-) \\
\hline CFY425 & JRY3009 MATa HMR-SS(abfl-) \\
\hline CFY474 & 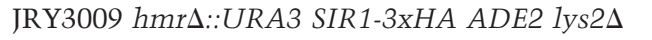 \\
\hline CFY476 & CFY474xCFY434 \\
\hline CFY629 & CFY474xCFY416 \\
\hline CFY633 & CFY474xCFY419 \\
\hline CFY632 & CFY474xCFY423 \\
\hline CFY625 & CFY474xCFY425 \\
\hline CFY687 & JRY3009 HMR-SS sir1-102-3xHA \\
\hline CFY689 & JRY3009 HMR-SS sir1-101-3xHA \\
\hline CFY105 & JRY3009 MATa HMR-SS SIR1-3xHA sir2A::LEU2 \\
\hline CFY762 & JRY3009 HMR-SS sir1A::LEU2 \\
\hline CFY914 & JRY3009 HMR-SS sir3 $\Delta:: L E U 2$ \\
\hline
\end{tabular}

deoxycholate, $1 \mathrm{mM}$ EDTA), and TE (20 mM Tris- $\mathrm{HCl}$ at $\mathrm{pH}$ 8.0, 1 mM EDTA), separated by SDS-polyacrylamide gel electrophoresis $(8 \%)$, and transferred to nitrocellulose. Sirlp-3xHA was detected with anti-HA antibodies. Protein-antibody complexes were visualized by enhanced chemiluminescence (Pierce SuperSignal DURA Detection System). To test whether Sir1p-3xHA had been quantitatively immunoprecipitated, we passed the supernatant fraction from an immunoprecipitation experiment through the immunoprecipitation protocol a second time, and the resulting immunoprecipitate was analyzed for Sirlp-3xHA.

To quantify the levels of Sirlp-3xHA in yeast, we compared the amount of Sirlp-3xHA immunoprecipitated from a crude extract or present in a chromatin fraction (see below) with a known amount of purified Ssq1-3xHA (Voisine et al. 2000).

Chromatin immunoprecipitation and analysis of immunoprecipitated DNA

The chromatin immunoprecipitation protocol used here was as described (Strahl-Bolsinger et al. 1997) except that $200 \mathrm{~mL}$ of yeast cells was grown in minimal media to a concentration of $2.0 \times 10^{7}$ cells $/ \mathrm{mL}$ before cross-linking, and, except for the experiments shown in Figures 6 and 8, cross-linking was performed for $60 \mathrm{~min}$. In addition, $125 \mathrm{mM}$ glycine and $2 \%$ ammonium hydroxide were added to quench the cross-linking reaction. The cells were harvested and broken as described above. The purified DNA from the immunoprecipitate was resuspended in $25 \mu \mathrm{L}$ TE. The purified DNA in the starting material (whole cell extract before immunoprecipitation) was resuspended in $1.0 \mathrm{~mL}$ TE.

To analyze for the presence of specific DNA fragments in the immunoprecipitate, we amplified the immunoprecipitated DNA by PCR using specific primers (Table 1). The PCRs were performed in $50 \mu \mathrm{L}$ with one-sixth of the immunoprecipitated material and $1 / 375$ th of the starting material. The number of PCR cycles yielding product within the linear range was determined empirically by analysis of twofold serial dilutions of the starting material. PCR products $(15 \mu \mathrm{L})$ were separated on $1 \%$ agarose gels and visualized with ethidium bromide.

To measure the association between Sirlp and $H M R$ at different stages of the cell cycle, we performed chromatin immu- noprecipitation experiments as described above except that 400 $\mathrm{mL}$ of yeast cells was grown to a concentration of $5 \times 10^{6}$ cells/ $\mathrm{mL}$ in rich media (YPD). The cells then were concentrated to $200 \mathrm{~mL}$ fresh YPD and allowed to continue growing asynchronously, or incubated with $0.2 \mathrm{M}$ hydroxyurea (S phase) or 10 $\mu \mathrm{g} / \mathrm{mL}$ nocodazole ( $\mathrm{M}$ phase) for $3 \mathrm{~h}$. To arrest cells in $\mathrm{G}_{1}$, we concentrated the cells to $40 \mathrm{~mL}$ and incubated them with $6 \mu \mathrm{M}$ $\alpha$-factor $\left(G_{1}\right.$ phase $)$ for $3 \mathrm{~h}$. When arrest was complete, the cells were diluted to $400 \mathrm{~mL}$ YPD and then incubated with formaldehyde. The yeast cells arrested with $>90 \%$ efficiency at all three stages as judged by cell morphology and cross-linking was performed for $15 \mathrm{~min}$.

\section{Nuclear and crude chromatin isolation}

To determine the approximate nuclear concentration of Sirlp$3 \mathrm{xHA}$ in sir2 $\Delta$ and sir3 $\Delta$ mutant strains, we isolated nuclei as described (Lue and Kornberg 1987) except the spheroplasts were washed on a sucrose cushion as described (Bell and Stillman 1992). Purified nuclei were resuspended in phosphate-buffered saline. Fifty $\mathrm{A}_{600}$ cell equivalents of isolated nuclei were used for nuclear immunoprecipitations.

Chromatin was isolated as described (Donovan et al. 1997) except that spheroplasts were prepared as described in the nuclear isolation procedure (Bell and Stillman 1992). The spheroplasts were harvested and washed two times with ice-cold wash buffer $\left(50 \mathrm{mM}\right.$ Tris at $\mathrm{pH} 7.5,20 \mathrm{mM} \mathrm{MgCl}_{2}, 100 \mathrm{mM}$ $\mathrm{NaCl}$ ). The cells were lysed on ice $20 \mathrm{~min}$ at a concentration of $250 \mathrm{~A}_{600}$ cell equivalents/mL in lysis buffer (wash buffer $+0.5 \%$ Triton X-100), and chromatin was isolated by centrifugation $\left(15,000 \mathrm{rpm}, 4^{\circ} \mathrm{C}\right.$ for $\left.5 \mathrm{~min}\right)$.

\section{Acknowledgments}

We thank Marc Gartenberg for providing pRS316-SIR3-3xHA and Rohinton Kamakaka for $\alpha-\mathrm{HA}$ used to begin this study and valuable advice and consultation during the course of this work. We also thank Jaerek Marszalek for purified and quantified Ssq1-3xHA protein. We are grateful to Michael Sheets and members of the Fox and Sheets laboratories for constructive criticism. This work was supported by a grant from the $\mathrm{Na}$ tional Institutes of Health (GM56890 to C.A.F.). K.A.G. was supported by a Biotechnology Training Grant to the University of Wisconsin-Madison (NIH 5T32 GM08349). C.A.F. is a recipient of a Burroughs Wellcome Fund Career Award in the Biomedical Sciences.

The publication costs of this article were defrayed in part by payment of page charges. This article must therefore be hereby marked "advertisement" in accordance with 18 USC section 1734 solely to indicate this fact.

\section{References}

Ansari, A. and Gartenberg, M.R. 1999. Persistence of an alternate chromatin structure at silenced loci in vitro. Proc. Natl. Acad. Sci. 96: 343-348.

Aparicio, O.M., Billington, B.L., and Gottschling, D.E. 1991. Modifiers of position effect are shared between telomeric and silent mating-type loci in S. cerevisiae. Cell 66: 12791287.

Bell, S.P. and Stillman, B. 1992. ATP-dependent recognition of eukaryotic origins of DNA replication by a multiprotein complex. Nature 357: 128-134.

Cheng, T.H. and Gartenberg, M.R. 2000. Yeast heterochromatin is a dynamic structure that requires silencers continuously. 
Genes \& Dev. 14: 452-463.

Chien, C.T., Buck, S., Sternglanz, R., and Shore, D. 1993. Targeting of SIR1 protein establishes transcriptional silencing at HM loci and telomeres in yeast. Cell 75: 531-541.

Donovan, S., Harwood, J., Drury, L.S., and Diffley, J.F. 1997. Cdc6-dependent loading of $\mathrm{Mcm}$ proteins onto pre-replicative chromatin in budding yeast. Proc. Natl. Acad. Sci. 94: 5611-5616.

Donze, D., Adams, C.R., Rine, J., and Kamakaka, R.T. 1999. The boundaries of the silenced HMR domain in Saccharomyces cerevisiae. Genes \& Dev.13: 698-708.

Eissenberg, J.C., Elgin, S.R., and Paro, R. 1995. Epigenetic regulation in Drosophila: A conspiracy of silence. In Chromatin structure and gene expression. (ed. S.C.R. Elgin), pp. 147171. Oxford University Press Inc., New York.

Foss, M., McNally, F.J., Laurenson, P., and Rine, J. 1993. A role of the origin recognition complex (ORC) in transcriptional silencing and DNA replication in Saccharomyces cerevisiae. Science 262: 1838-1844.

Fox, C.A., Loo, S., Rivier, D.H., Foss, M., and Rine, J. 1993. A transcriptional silencer as a specialized origin of replication that establishes functional domains of chromatin. Cold Spring Harb. Symp. Quant. Biol. 58: 443-455.

Fox, C.A., Loo, S., Dillin, A., and Rine, J. 1995. The origin recognition complex has essential functions in transcriptional silencing and chromosomal replication. Genes \& Dev. 9: $911-924$

Fox, C.A., Ehrenhofer-Murray, A.E., Loo, S., and Rine, J. 1997. The origin recognition complex, SIR1, and the S phase requirement for silencing. Science 276: 1547-1551.

Freeman-Cook, L.L., Sherman, J.M., Brachmann, C.B., Allshire, R.C., Boeke, J.D., and Pillus, L. 1999. The Schizosaccharomyces pombe hst4(+) gene is a SIR2 homologue with silencing and centromeric functions. Mol. Biol. Cell 10: $3171-3186$.

Gailus-Durner, V., Xie, J., Chintamaneni, C., and Vershon, A.K. 1996. Participation of the yeast activator Abf 1 in meiosisspecific expression of the HOP1 gene. Mol. Cell. Biol. 16: $2777-2786$.

Gardner, K.A., Rine, J., and Fox, C.A. 1999. A region of the Sir1 protein dedicated to recognition of a silencer and required for interaction with the Orcl protein in Saccharomyces cerevisiae. Genetics 151: 31-44.

Gottlieb, S. and Esposito, R.E. 1989. A new role for a yeast trasncriptional silencer gene, SIR2, in regulation of recombination in ribosomal DNA. Cell 56: 771-776.

Gottschling D.E., Aparicio, O.M., Billington, B.L., and Zakian, V.A. 1990. Position effect at S. cerevisiae telomeres: Reversible repression of Pol II transcription. Cell 63: 751-762.

Grunstein, M. 1997. Molecular model for telomeric heterochromatin in yeast. Curr. Opin. Cell Biol. 9: 383-387.

Guthrie, C. and Fink, G.R. 1991. Guide to yeast genetics and molecular biology. Academic Press, San Diego, CA.

Hecht, A., Laroche, T., Strahl, B.S., Gasser, S.M., and Grunstein, M. 1995. Histone H3 and H4 N-termini interact with SIR3 and SIR4 proteins: A molecular model for the formation of heterochromatin in yeast. Cell 80: 583-592.

Hecht, A., Strahl-Bolsinger, S., and Grunstein, M. 1996. Spreading of transcriptional repressor SIR3 from telomeric heterochromatin. Nature 383: 92-96.

Hendrich, B.D. and Willard, H.F. 1995. Epigenetic regulation of gene expression; the effect of altered chromatin structure from yeast to mammals. Hum. Mol. Gen. 4: 1765-1777.

Henikoff, S. 2000. Heterochromatin function in complex genomes. Biochim. Biophy. Acta 1470: 1-8.

Herskowitz, I., Rine, J., and Strathern, J. 1992. Mating-type de- termination and mating-type interconversion in Saccharomyces cerevisiae. In The molecular and cellular biology of the yeast Saccharomyces cerevisiae: Gene expression (ed. E. Jones, J.R. Pringle, and J.R. Broach), pp. 583-656. Cold Spring Harbor Laboratory Press, Cold Spring Harbor, New York.

Holmes, S.G. and Broach, J.R. 1996. Silencers are required for inheritance of the repressed state in yeast. Genes \& Dev. 10: 1021-1032.

Huang, D.W., Fanti, L., Pak, D.T.S., Botchan, M.R., Pimpinelli, S., and Kellum, R. 1998. Distinct cytoplasmic and nuclear fractions of Drosophila heterochromatin protein 1: Their phosphorylation levels and associations with origin recognition complex proteins. J. Cell Biol. 142: 307-318.

Imai, S., Armstrong, C.M., Kaeberlein M., and Guarente, L. 2000. Transcriptional silencing and longevity protein Sir2 is a NAD-dependent histone deacetylase. Nature 403: 795800.

Kang, J.J., Yokoi, T.J., and Holland, M.J. 1995. Binding sites for abundant nuclear factors modulate RNA polymerase I-dependent enhancer function in Saccharomyces cerevisiae. I. Biol. Chem. 270: 28723-28732.

Kirschmann, D.A., Lininger, R.A., Gardner, L.M., Seftor, E.A., Odero, V.A., Ainszten, A.M., Earnshaw, W.C., Wallrath, L.L., and Hendrix, M.J. 2000. Down-regulation of HP1Hsalpha expression is associated with the metastatic phenotype in breast cancer. Cancer Res. 60: 3359-3363.

Landry, J., Sutton, A., Tafrov, S.T., Heller, R.C., Stebbins, J., Pillus, L. and Sternglanz, R. 2000. The silencing protein SIR2 and its homologs are NAD-dependent protein deacetylases. Proc. Nat1. Acad. Sci. 97: 5807-5811.

Liang, C., Weinreich, M., and Stillman, B. 1995. ORC and Cdc6p interact and determine the frequency of initiation of DNA replication in the genome. Cell 81: 667-676.

Loo, S. and Rine, J. 1994. Silencers and domains of generalized repression. Science 264: 1768-1771.

- 1995. Silencing and domains of heritable gene expression. Annu. Rev. Cell Dev. Biol. 11: 519-548.

Loo, S., Fox, C.A., Rine, J., Kobayashi, R., Stillman, B., and Bell, S. 1995. The origin recognition complex in silencing, cellcycle progression, and DNA replication. Mol. Biol. Cell 6: 741-756.

Lue, N.F. and Kornberg, R.D. 1987. Accurate initiation at RNA polymerase II promoters in extracts from Saccharomyces cerevisiae. Proc. Nat1. Acad. Sci. 84: 8839-8843.

Mahoney, D.J., Marquardt, R., Shei, G.J., Rose, A.B., and Broach, J.R. 1991. Mutations in the HML-E silencer of Saccharomyces cerevisiae yield metastable inheritance of transcriptional repression. Genes \& Dev. 5: 605-615.

Marahrens, Y. and Stillman, B. 1992. A yeast chromosomal origin of DNA replication defined by multiple functional elements. Science 255: 817-823.

McNally, F.J. and Rine, J. 1991. A synthetic silencer mediates SIR-dependent functions in Saccharomyces cerevisiae. Mol. Cell. Biol. 11: 5648-5659.

Micklem, G., Rowley, A., Harwood, J., Nasmyth, K., and Diffley, J.F. 1993. Yeast origin recognition complex is involved in DNA replication and transcriptional silencing. Nature 366: 87-89.

Miller, A.M. and Nasmyth, K.A. 1984. Role of DNA replication in the repression of silent mating type loci in yeast. Nature 312: 247-251.

Moretti, P., Freeman, K., Coodly, L., and Shore, D. 1994. Evidence that a complex of SIR proteins interacts with the silencer and telomere-binding protein RAP1. Genes \& Dev. 8: 2257-2269.

Pak, D.T.S., Pflumm, M., Chesnokov, I., Huang, D.W., Kellum, 
R., Marr, J., Romanowski, P., and Botchan, M.R. 1997. Association of the origin recognition complex with heterochromatin and HP1 in higher eukaryotes. Cell 91: 311-323.

Palacios DeBeer, M.A. and Fox, C.A. 1999. A role for a replicator dominance mechanism in silencing. EMBO $J$. 18: $3808-3819$.

Pillus, L. and Rine, J. 1989. Epigenetic inheritance of transcriptional states in S. cerevisiae. Cell 59: 637-647.

Pillus, L. and Grunstein, M. 1995. Chromatin structure and epigenetic regulation in yeast. In Chromatin structure and gene expression. (ed. S.C.R. Elgin), pp. 123-146. Oxford University Press, New York.

Quintana, D.G. and Dutta, A. 1999. The metazoan origin recognition complex. Front. Biosci. 4: 805-815.

Reed, S.H., Akiyama, M., Stillman, B., and Friedberg, E.C. 1999. Yeast autonomously replicating sequence binding protein is involved in nucleotide excision repair. Genes \& Dev. 13: 3052-3058.

Rolfes, R.J., Zhang, F., and Hinnebusch, A.G. 1997. The transcriptional activators BAS1, BAS2, and ABF1 bind positive regulatory sites as the critical elements for adenine regulation of ADE5,7. J. Biol. Chem. 272: 13343-13354.

Schneider, B.L., Seufert, W., Steiner, B., Yang, Q.H., and Futcher, A.B. 1995. Use of polymerase chain reaction epitope tagging for protein tagging in Saccharomyces cerevisiae. Yeast 11: 1265-1274.

Shore, D. 1994. RAP1: A protean regulator in yeast. Trends Genet. 10: 408-412.

Smith, J.S., Brachmann, C.B., Celic, I., Kenna, M.A., Muhammad, S., Starai, V.J., Avalos, J.L., Escalante-Semerena, J.C., Grubmeyer, C., Wolberger, C., and Boeke, J.D. 2000. A phylogenetically conserved NAD+ dependent protein deacetylase activity in the Sir2 protein family. Proc. Natl. Acad. Sci. 97: 6658-6663.

Stevenson, J.B. and Gottschling, D.E. 1999. Telomeric chromatin modulates replication timing near chromosome ends. Genes \& Dev. 13: 146-151.

Strahl-Bolsinger, S., Hecht, A., Luo, K., and Grunstein, M. 1997. Sir2 and Sir4 interactions differ in core and extended telomeric heterochromatin in yeast. Genes \& Dev. 11: 83-93.

Tanny, J.C., Dowd, G.J., Huang, J., Hilz, H. and Moazed, D. 1999. An enzymatic activity in the yeast Sir2 protein that is essential for gene silencing. Cell 99: 735-745.

Terleth, C., van Sluis, C.A., and van de Putte, P. 1989. Differential repair of UV damage in Saccharomyces cerevisiae. Nucleic Acids Res. 17: 4433-4439.

Terleth, C., Schenk, P., Poot, R., Brouwer, J., and van de Putte, P. 1992. Differential repair of UV damage in rad mutants of Saccharomyces cerevisiae: A possible function of G2 arrest upon UV irradiation. Mol. Cell. Biol.10: 4678-4684.

Triolo, T. and Sternglanz, R. 1996. Role of interactions between the origin recognition complex and Sirl in transcriptional silencing. Nature 381: 251-253.

Voisine, C., Schilke, B., Ohlson, M., Beinert, H., Marszalek, J., and Craig, E.A. 2000. Role of the mitochondrial Hsp70s, Ssc1 and Ssq1, in the maturation of Yfh1. Mol. Cell. Biol. 20: $3677-3684$. 


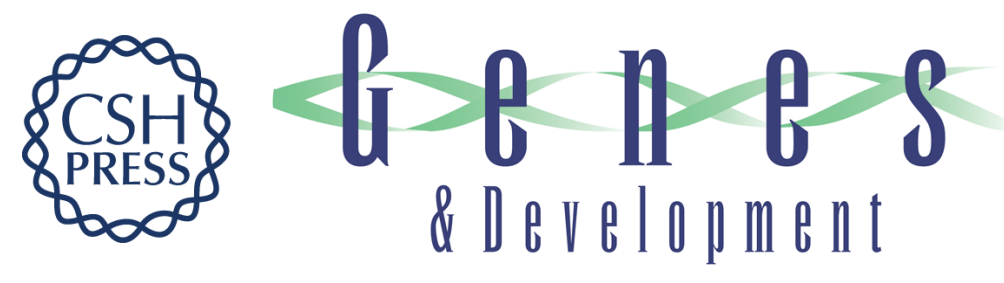

\section{The Sir1 protein's association with a silenced chromosome domain}

Kelly A. Gardner and Catherine A. Fox

Genes Dev. 2001, 15:

Access the most recent version at doi:10.1101/gad.852801

References This article cites 54 articles, 29 of which can be accessed free at: http://genesdev.cshlp.org/content/15/2/147.full.html\#ref-list-1

License

Email Alerting Receive free email alerts when new articles cite this article - sign up in the box at the top Service right corner of the article or click here.

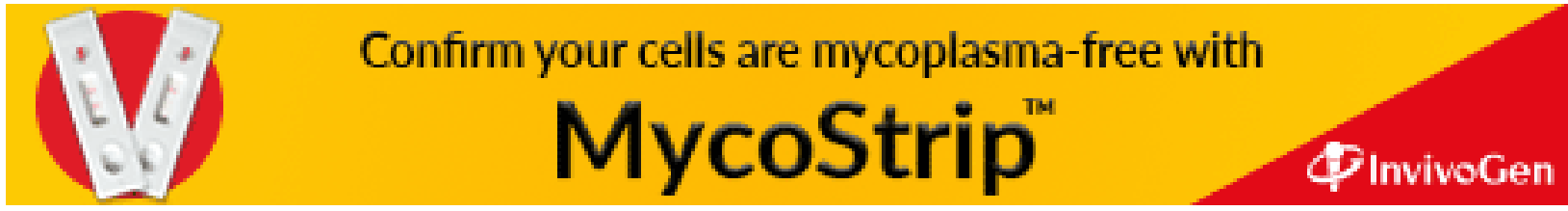

